\title{
Lights, camera...
}

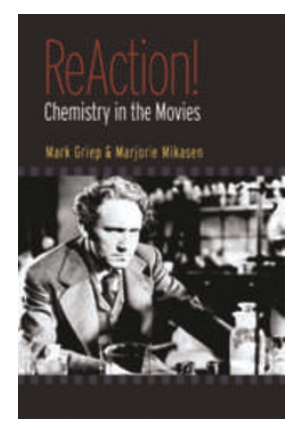

ReAction!

Chemistry in the

Movies

by Mark A. Griep and

Marjorie L. Mikasen

OXFORD UNIV. PRESS:

2009. 350 PP. $€ 32.50$

$\mathrm{f}$ society can be reflected in its culture, and movies are now the most popular form of culture, what can science in the movies tell us about science in the real world? This is the idea behind ReAction! Chemistry in the Movies by Mark Griep and Marjorie Mikasen. It is far more than a dry discussion of the when, what, where and how of chemistry in all its forms in the movies: over ten chapters the authors examine how chemistry and chemists are portrayed on film.

Beginning with the most obvious example of Jekyll and Hyde, each chapter looks at a particular theme shared by chemistry and film. The first five are 'negative' themes and the last five are 'positive'. Within each chapter the theme is discussed, the chemistry expanded, and then placed in broad psychological, social, historical and cinematic context. Finally the movies that best represent the theme are described. In the introduction the authors describe how the book uses the Jekyll and Hyde concept - each 'dark' chapter has its 'light' partner. And the topic of reflection reappears regularly as a clever, if occasionally forced, device to hold the themes together.

This division into themes, although arguably slightly artificial, works well, allowing the discussion to move across broad areas - from the Jekyll and Hyde split personality through invisibility, chemical warfare, the chemical industry, pollution and drugs in the 'dark' chapters to inventors, forensics, scientific testing, medicinal chemistry and scientific discovery in the 'light' chapters.

Many of the stories behind the science have been told elsewhere, but are no less interesting for that, and the authors narrate them well. The discussion around themes in the movies and in chemistry highlights some interesting ideas and comparisons. As noted above, the discussion does occasionally feel forced - as if the authors wanted to include certain ideas, but couldn't always fit them to the theme. And, in several places the book drifts off topic entirely - the discussion of terrorism and the US response in the chapter on chemical warfare, for example, feels overtly political and out of place. At other times, however, the discussion is very well done - the reader doesn't notice the various digressions from the theme and is pleasantly surprised to find the narrative returning gently to the movies and the chemistry.

Strangely, the least integrated parts are the descriptions of the films at the end of each chapter. Each description features an archetypal film described in some detail - and in the context of the theme followed by several short synopses of other exemplifying films. These can feel tacked on to the end, and their purpose isn't always clear. It is possible that these are here to help teachers use the book as a teaching aid, rather than for the casual reader, or are there to assist the reader in tracking down some less well known pictures.

In fact, the attempt to create a popular science book and teaching aid (the first appendix is about how to use this book in the classroom) is perhaps one of the weaknesses of the book. The target audience is often unclear - a novice scientist could be put off by some of the descriptions of the science and a more confident scientist might find some of it overly simple.

The book, then, like its main example Dr Jekyll, suffers from a split personality. For the most part the ideas and writing are fascinating and engrossing, but just occasionally it lapses into being awkward and forced. In the end, though, the book leaves the reader with plenty of ideas for films to see and some excellent stories about chemists and chemistry. But more than that it presents a fascinating view of chemistry, the movies and chemistry in the movies, which gives the reader plenty of food for thought next time a bit of chemistry appears on the silver screen; and this is perhaps far more than one might expect from a book about chemistry in the movies.

\section{REVIEWED BY EDWARD MORGAN}

Edward Morgan is a film fan and in his spare time is a Publishing Editor for the Royal Society of Chemistry.

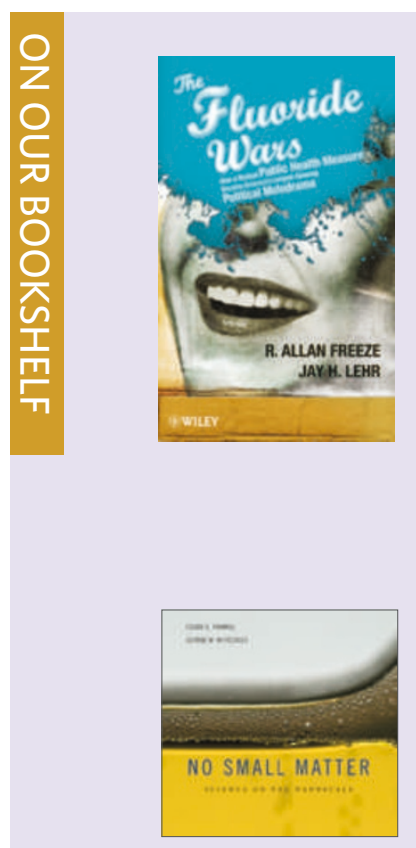

The Fluoride Wars: How a Modest Public Health Measure Became America's Longest-Running Political Melodrama

By R. Allen Freeze and Jay H. Lehr WILEY: 2009. 383 PP. $€ 26.95$

This is the story of the long-standing dispute between those in favour of fluoridation of drinking water and those against it. An unusual book on this topic, it tries to present both sides of the argument in a balanced fashion ultimately concluding that the two sides need to talk to one another. Outside the obvious interest to those in the field, the book gives a glimpse of what happens when science and politics collide.

\section{No Small Matter: Science on the Nanoscale} By Felice C. Frankel and George M. Whitesides HARVARD UNIV. PRESS: 2009. 192 PP. $€ 25.95$

Big pictures describe small things in this second book collaboration by noted science photographer Felice Frankel and chemist George Whitesides. The book meanders through a broad swathe of topics from the internet to ocean sponges to wave-particle duality, accompanied by stunning images and easy to understand (if occasionally whimsical) prose. Tricky science is made both simple and pretty, making the book stimulating for scientist and non-scientist alike. 